\title{
Modelo de gestión de conocimiento para un Joint-Venture sistemático entre la Facultad Tecnológica de la Universidad Distrital y el sector del software
}

\author{
Knowledge management model for a systematic Joint-Venture between \\ Technological Faculty of Distrital University and software business
}

Luis A. Rojas Castellar ${ }^{*}$

Juan C. Guevara Bolaños**

Ginna Largo Ordóñez ${ }^{* * *}$

\section{RESUMEN}

La interacción universidad-empresa se ha hecho necesaria para incrementar la productividad de ambos agentes en la sociedad. Sin embargo, suelen trabajar en sentidos contrarios: la universidad se encarga de la producción del conocimiento mediante la investigación e innovación; mientras que las empresas implantan la tecnología dentro de los procesos productivos y comercializan artículos y servicios en el mercado. Si bien ambos agentes tienen necesidades complementarias, en el caso colombiano no existe una unión semántica. En consecuencia, este artículo pretende construir un modelo de gestión que articule el conocimiento con las necesidades, tanto universitarias como empresariales, para así maximizar el beneficio de la sociedad como conjunto. Así mismo, expone el avance parcial en el desarrollo y la evaluación de un modelo piloto -prototipode cooperación entre la Facultad Tecnológica de la Universidad Distrital ${ }^{1}$ y algunas empresas del sector del software en Bogotá D.C. (Colombia) -implementado por medio de la construcción de un portal web. La lectura de éste trabajo da cuenta de experiencias basadas en el acercamiento mutuo entre universidades y diferentes sectores productivos del país.

\section{Palabras clave}

Joint-ventures, universidad-empresa, modelos de gestión de conocimiento, sistemas nacionales de innovación, crecimiento económico, industria del software en Colombia.

* Ingeniero Electricista y Magister en Economía de la Universidad Nacional de Colombia. Docente de Planta en la Facultad Tecnológica de la Universidad Distrital. Grupo de Investigación Metis - Líder Línea de Cooperación Universidad Empresa, larojasc@udistrital. edu.co.

** Docente de Planta, Director de la Unidad de Investigación y del Grupo de Investigación Metis de la Facultad Tecnológica de la Universidad Distrital, jcguevarab@udistrital.edu.co.

*** Tecnóloga en Sistematización de Datos y estudiante de Ingeniería Telemática en la Facultad Tecnológica de la Universidad Distrital. Coordinadora del Semillero de Investigación Pegasus e integrante del Grupo de Investigación Metis, ginna_largo_ud@yahoo.com.

1 Este artículo es un avance parcial del proyecto de investigación titulado Formulación y evaluación de un modelo de gestión de conocimiento para el desarrollo de la interacción universidad-empresa, de la Facultad Tecnológica de la Universidad Distrital. Este proyecto es financiado por el centro de Investigaciones y Desarrollo Científico de la Universidad Distrital (Cidc). 


\section{ABSTRACT}

Nowadays, University-Industries interaction is necessary in order to increase productivity for both agents. However, they use to work in different sides: Universities as knowledge production core throughout research and innovation. Industries apply technology and take products and services in market environment. However, even when these two agents have complementary needs, they do not use to work together, at least in the Colombian case. In this way, a knowledge management model is needed which connects universities and firms in order to maximize social welfare. This article shows the development, application an evaluation of a prototype model with mutual cooperation between Technological Faculty from Universidad Distrital and Colombia's software industries. The practical application was developed throughout a web page. Different lessons of systematic approach between universities and other Colombias industries can be learnt due to this work.

\section{Key words}

University-industries, Joint-Ventures, knowledge management model, national innovation systems, economic growth, Colombia's software industry.

\section{INTRODUCCIÓN}

Los procesos de innovación y desarrollo tecnológico constituyen uno de los métodos más frecuentes para incrementar el valor agregado de un producto en las empresas; son un elemento diferenciador que puede hacer parte de la estrategia utilizada para vencer a la competencia (Vargas, 2003). Desafortunadamente, en muchas firmas, los procesos de innovación representan un alto riesgo y no suelen producir resultados en corto plazo (las compañías no están preparadas para llevarlos a cabo). En consecuencia, algunas empresas suelen recurrir a estrategias de competitividad basadas en la reducción de costos pero, como explica Porter (1987), no son efectivas a largo plazo porque los compe- tidores también alcanzan ese logro con facilidad $\mathrm{y}$, entonces, retornan al escenario de rendimientos decrecientes. No obstante, existe una estrategia que minimiza el riesgo de aventurarse en la industria e incentiva la innovación tecnológica: aliarse a una institución especializada en el desarrollo de proyectos de investigación y crecimiento. Dicha institución es la universidad, cuya finalidad máxima es la producción y difusión de conocimiento, pero cuyo inconveniente usualmente radica en la dificultad de trasladar sus logros a los procesos productivos reales y al mercado. De ésta manera, es evidente que tanto universidades como empresas poseen necesidades complementarias y que una estructura de colaboración mutua tipo Joint Venture $^{2}$ puede maximizar el beneficio para la sociedad (Corona, 2008); empero, nuestro país carece de una estructura sistemática que integre estos intereses y concilie sus diferencias existentes. Karthikeyan (2008) expone que en India se estableció una interacción exitosa entre estudiantes de ingeniería y empresas de tecnologías de información -por medio de políticas interinstitucionales- que ha sido materializada a través de convenios concretos. Entre tanto, para el caso colombiano, puede ser posible una integración exitosa de este par de agentes siempre y cuando se formulen marcos institucionales y sistemáticos que los acerquen.

De acuerdo con este diagnóstico, el desarrollo de procesos sistemáticos de acercamiento entre universidades y empresas es necesario. Éste proyecto representa un prototipo de cooperación entre la Facultad Tecnológica de la Universidad Distrital con empresas del sector del software en Bogotá D.C. y desarrolla un modelo de gestión de conocimiento (implementado por medio de un portal web) que le permite a los proyectos de grado y de investigación desarrollados en las universidades ${ }^{3}$

2 Joint venture es un tipo de alianza entre dos o más instituciones en busca de desarrollar unos objetivos comunes. Usualmente está referido a acuerdos comerciales de inversión conjunta entre empresas aunque, en un contexto más amplio, pueden incluirse diferentes instituciones como universidades, gobiernos, representaciones gremiales, etc.

3 Dentro del proyecto piloto - prototipo- únicamente serán publicados los proyectos de grado e investigación producidos por el Grupo de Investigación Metis de la Facultad Tecnológica (Universidad Distrital). Sin embargo, para 
estar disponibles para el conocimiento de las empresas - por parte de éstas serán publicadas ofertas de proyectos de cooperación mutua en investigación.

\section{ESTADO DEL ARTE: INTERACCIÓN UNIVER- SIDAD-EMPRESA}

\section{Contexto internacional}

Las universidades de los principales países desarrollados se han enfocado, en los últimos años, en interactuar más a fondo con la industria. En efecto, el Reino Unido, China y Australia; entre otros, han instaurado, para los proyectos de investigación desarrollados en las universidades, programas de evaluación permanentes y rigurosos con el fin de asegurar que los resultados de dichas instituciones tengan alto impacto, exhiban datos concretos y se implementen en la industria (Geuna, 2003). Éstos hechos están sustentados en una política desarrollada especialmente por Estados Unidos mediante las leyes Stevenson Wylder (1980) y Bay Dohle (1986), que permiten a universidades y profesores trabajar -en proyectos financiados por fondos federales-, patentar y obtener regalías si alguna innovación es exitosa y rentable en el mercado. Incluso, las regulaciones exigen a los laboratorios financiados a transferir la tecnología desarrollada hacia el sector privado. De forma similar, el Reino Unido desarrolló un seguimiento institucional en el cual la tecnología desarrollada en las universidades debe aplicarse al sector productivo por medio de un instituto de carácter nacional encargado del monitoreo y la evaluación de los proyectos de investigación (Higher Education Funding Councils). En el resto de Europa, durante los primeros años del siglo XXI, según Manjarrés (2008), la interacción universidad-empresa todavía se encuentra desacoplada y cada agente se preocupa más por sus propios intereses: las universidades producen conocimiento y lo publican mientras que las empresas aplican tecnología, comercializan sus pro-

proyectos futuros se pretende que la base de datos del aplicativo contenga todos los proyectos desarrollados en la universidad clasificados por: facultad, carrera, proyecto curricular y grupo de investigación. ductos y servicios y mantienen como secreto industrial las innovaciones desarrolladas al interior de las mismas. En general, el modelo anglosajón de innovación dentro de las universidades se preocupa porque ésta tenga una utilidad concreta dentro del sector productivo, mientras que en modelos más clásicos como Italia y Francia no existe una presión hacia las universidades para que sus innovaciones sean realmente usadas dentro de ambientes industriales.

Luego de apreciar el actual escenario internacional, es indiscutible que los agentes universidad y empresas no se atraen por sí mismas. Por ende, se necesita de un escenario institucional para articularlas.

\section{Contexto nacional}

Si se efectúan comparaciones en cuanto a la innovación de América Latina con la de Estados Unidos -o algunos países europeos- se obtiene una conclusión tan básica como obvia: la innovación en los países en desarrollo es muy pobre; además, ésta condición se corrobora ya que el índice de innovación -mide los logros de investigación mediante el número de artículos publicados, profesores con doctorado y número de patentes (Warner, 2000) - es, en promedio para América Latina, de -0,99 respecto al 2 de Estados Unidos4 (por su parte, los datos de Colombia se encuentran ligeramente superiores al promedio). Ahora bien, estos indicadores, aparte de relacionarse directamente con innovaciones radicales y susceptibles de patentar, no consideran las de tipo simple o incremental (éstas últimas son más susceptibles de generarse y necesitan menos cantidad de recursos). En base a lo anterior, Colombia debería basar su estrategia de innovación -y de cooperación universidad-empresa- en la adaptación de procesos y productos tecnológicos ya existentes cuyas optimizaciones fueran significativas al medio nacional y más económicas respecto a los mismos avances incrementales importados. De acuerdo a esto, y rea-

4 El índice de innovación es un indicador adimensional que oscila entre -2 y 2 . Reúne varios aspectos de la innovación como el número de patentes, artículos publicados, profesores con doctorado y otros aspectos (transferencia y difusión de la tecnología). 
lizando un análisis sectorial, es evidente que una de las pocas áreas factibles para que el país desarrolle innovaciones de ésta índole -incluso de tipo radical con un altísimo valor agregado- es el software; la infraestructura existente en Colombia se encuentra en igualdad de condiciones en comparación a la de los países desarrollados. La única variable que hace la diferencia es el talento intelectual, lo cual puede aprovecharse y organizarse en modelos de gestión exitosos a través de esquemas similares a los de Irlanda, India y Brasil.

\section{INDUSTRIA DEL SOFTWARE EN COLOMBIA}

\section{Análisis sectorial}

Una de las técnicas mejor elaboradas para determinar las ventajas competitivas entre empresas es el Análisis de Michael E. Porter (Porter, 1998), el cual se centra en los siguientes aspectos: amenaza de entrada de nuevos competidores, rivalidad entre competidores, poder de negociación de proveedores, poder de negociación de compradores y amenaza de ingreso de productos sustitutos. El Instituto Tecnológico de Massachusetts “MIT" (Cepero, 2007), donde se aplicó un criterio modificado de Porter para comparar diferentes sectores de la economía, concluyó que la industria del software es estratégica en Colombia -especialmente para clientes del sector bancario, financiero, empresarial y gubernamental. En cuestión de proveedores de recurso humano calificado, la producción de software es intensiva en la mano de obra y, por lo tanto, necesita de gran cantidad de personas con fuertes habilidades en programación y en el manejo del idioma inglés. Por ejemplo, India, el exportador de software número uno del mundo, posee alrededor de un millón y medio de programadores (Nezu, 2005). De esta manera, la universidad puede interactuar de forma fluida con el sector del software mediante del ofrecimiento del recurso humano calificado.

\section{Situación del sector del software en Colombia}

Según la Cámara de Comercio de Bogotá, en Colombia existen alrededor de 4000 empresas de
FIGURA 1. Factores de dificultad en la competitividad de las empresas de software en Colombia.

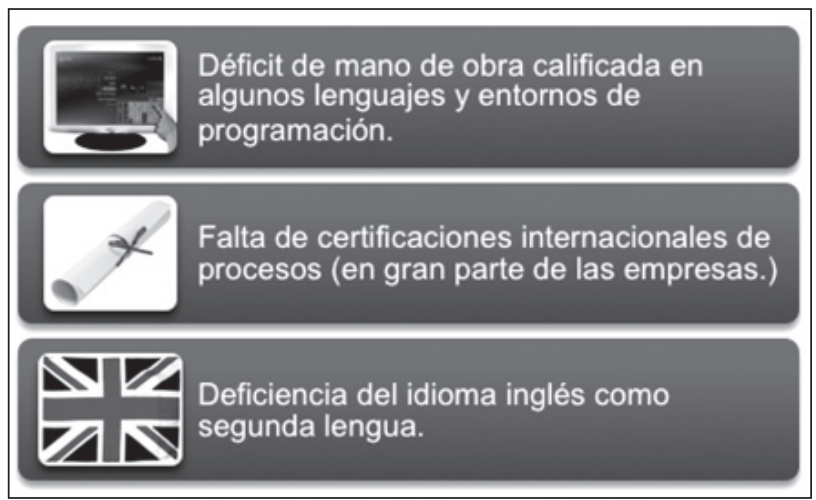

software dedicadas fundamentalmente a la venta e instalación de licencias, prestación de servicios de consultoría en tecnologías de la información y desarrollo de software enfocado hacia el mercado local. La exportación, si bien pareciera una muy buena alternativa para éste sector, se encuentra en descenso; en 2008 se presentaron ventas por USD\$ 4 millones, representando un fuerte decrecimiento si se compara con los USD \$ 25 millones de 20015. Sin embargo, aún cuando éste indicador presume un declive notorio en la industria del software en Colombia y si se tiene en cuenta que países líderes como India e Irlanda sustentan sus ingresos casi en un $90 \%$ en exportaciones, no parece tan grave; Brasil, líder de la industria en América Latina, está orientado en un $90 \%$ hacia su mercado interno. Los inconvenientes que desestimulan el interés de las empresas de software (figura 1) por el mercado externo tienen su explicación en los sistemas tributario y de divisas. Básicamente, para las industrias del software, no es rentable tributar, además de Colombia, en el país destino de la exportación del software -en particular por la ausencia de tratados comerciales que excluyen al sector de éstas condiciones desfavorables.

Existen características en el mercado interno colombiano que hacen de la industria del software un sector dinámico y progresivo. Algunas de las más importantes son: el incremento de automatización

5 Ésta información fue suministrada por Fedesoft, organización que representa a las empresas de software en Colombia.

REVISTA CIENTÍFICA / ENERO -DICIEMBRE DE 2010 / No. 12 / BOGOTÁ, D.C. 
de procesos en la industria y el sector financiero, el desarrollo significativo en la cobertura y en la calidad de los servicios de banda ancha, y la existencia de un mercado formal de expedición de licencias de software.La interacción universidad-empresa, entre el sector del software y la Facultad Tecnológica de la Universidad Distrital, puede sustentarse, básicamente, en la oferta de mano de obra calificada en servicios especializados -dada la escasez de personal en algunos lenguajes y entornos de la programación. En consecuencia, puede proponerse el desarrollo de trabajos de colaboración mutuos, ya sean cofinanciados como proyectos de investigación o de manera libre a través de proyectos de grado. Una gran ventaja para la interacción entre la facultad y las empresas de software es que, además del interés de trabajar en equipo, no se necesitan grandes infraestructuras más allá de una buena cobertura de banda ancha.

\section{MODELO DE GESTIÓN DE CONOCIMIENTO ENTRE LA FACULTAD TECNOLÓGICA Y LAS EMPRESAS DE SOFTWARE}

\section{Marco histórico}

Los modelos de gestión de conocimiento nacieron como consecuencia del trabajo desarrollado por Nonaka y Takeuchi (Nonaka, 1995), luego de que analizaran el comportamiento de los flujos de conocimiento al interior de las empresas japonesas. Los investigadores detectaron dos tipos de conocimiento: explícito y tácito. El primero se encuentra disponible en la literatura científica convencional y es fácilmente transferible a los individuos en general; por su parte, el segundo está relacionado a la experiencia y la pericia (prácticas difíciles de transferir de un individuo a otro). El modelo se encuentra basado en el funcionamiento de las empresas japonesas, así que no podría gozar de mucha validez en entornos industriales internacionales. No obstante, es lo suficientemente robusto para originar otros. Entre éstos últimos se encuentran los prototipos de Quinn, Anderson y Finkelstein (Quinn, 1996), usados en hospitales, firmas de consultoría y hoteles; indican el comportamiento de organizaciones invertidas donde los flujos de conocimiento trascurren desde los empleados -vendedores- que están en contacto directo con los consumidores hasta el gerente o un alto directivo de la compañía. Dada la inexistencia de un modelo único de gestión de conocimiento, empresas como Sony, Toshiba, McDonalds y British Petroleum (Krogh, 2000) han desarrollado modelos que representan sus propios escenarios industriales. Conforme a lo anterior, cada marco industrial tiene la libertad de representar el modelo de gestión de conocimiento que más se adapte a las condiciones particulares del entorno analizado, pues los modelos teóricos, como el de Nonaka, constituyen apenas una guía metodológica. Incluso, la diversidad de modelos es notable aún en la estructura europea Ekmf (European Knowledge Managment Framework) que, si bien intenta unificar la terminología respecto de modelos de gestión de conocimiento, ratifica la existencia de infinidad de ellos de acuerdo a cada escenario particular. Según ésta situación, se planteará un modelo particular para la interacción entre las empresas de software y la Facultad Tecnológica de la Universidad Distrial Francisco José de Caldas.

\section{Modelo de gestión de conocimiento: Facultad Tecnológica (UD) - empresas de software}

El modelo planteado (figura 2) fue diseñado siguiendo una estructura de correlación entre ofertas y demandas -con base en la teoría económica (Jehle, 2001). En la interrelación de varios agentes, cada uno demanda la solución de alguna necesidad en particular y ofrece productos y servicios. Así mismo, existen algunas parejas en donde las ofertas de un agente satisfacen las demandas de otro (en estos casos se propone la hipótesis del interés por mantener una relación de largo plazo, pues ambas partes estarán satisfechas). Para el caso de la interacción entre la Facultad Tecnológica y las empresas de software, se elaboró una lista de ofertas y demandas pertenecientes a cada uno de los agentes y se determinaron los puntos en donde existe correlación. 


\section{FIGURA 2. Modelo de gestión de conocimiento Facul- tad Tecnológica-empresas de software.}

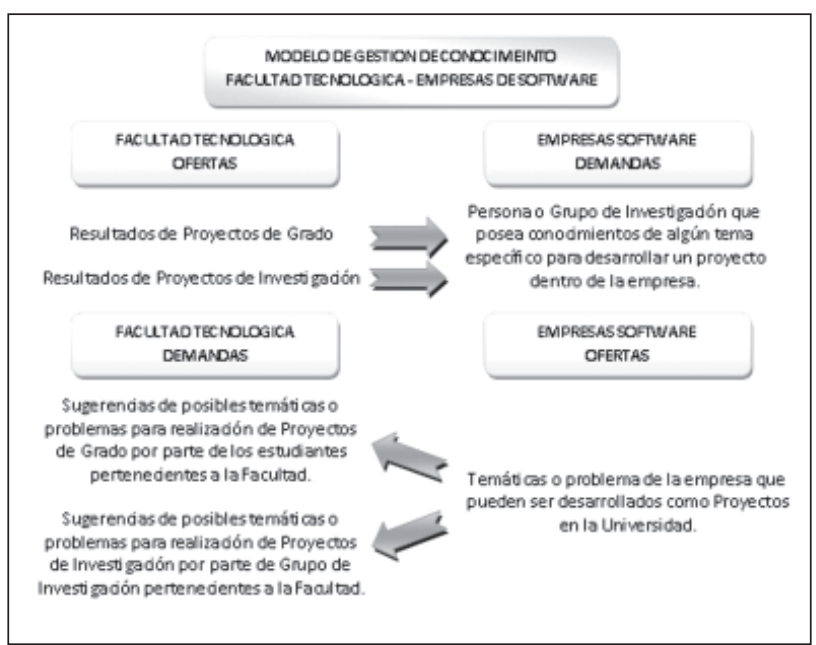

Una de las demandas o requerimientos comúnmente expresada por las empresas de software es la búsqueda de personal calificado en alguna temática en particular; por ejemplo: conocimientos en software especializados como PI server, Matlab, etc. En éstos casos no es fácil encontrar personal de alto nivel calificado o programadores que tengan el conocimiento de algún tema específico y, entonces, las empresas emprenden la búsqueda a nivel de consultoría -lo cual se traduce en altos costos. Por otro lado, la Facultad Tecnológica, y en general cualquier universidad, desarrolla en su interior una cantidad considerable de proyectos de grado e investigaciones sobre temas diversos (incluyendo algunos bastante especializados). En repetidas ocasiones, los resultados de estos proyectos son desconocidos por el mercado debido a inconvenientes relacionados con la difusión insuficiente de los mismos. De acuerdo a este escenario, se observa que puede existir una correlación entre la demanda de las empresas de software -de personal especializado en temas muy específicos-, la oferta de proyectos de grado y las investigaciones que pueden haber trabajado en ese tema particular (cabe anotar que ésta relación depende de la existencia de una gran cantidad de proyectos en la base de datos y de la regularidad en las actualizaciones). De esta manera, en el modelo planteado, las empresas, mediante el aplicativo en Internet que se desarrolló, digitan el tema en el que necesitan colaboración e inmediatamente se realiza una búsqueda masiva dentro de los proyectos de grado e investigaciones desarrolladas en la facultad. En los resultados aparece una lista con las coincidencias efectuadas y los datos específicos del autor del proyecto, sea un estudiante o un grupo de investigación. La empresa, entonces, puede comunicarse con las personas indicadas para solicitar la consultoría o asesoría necesaria en el tema requerido.

Entre tanto, los estudiantes, la mayoría de las veces, desconocen la existencia de empresas interesadas en trabajar y financiar proyectos; hecho que hace necesario, por parte de la universidad, la creación de un banco de datos o ideas del sector industrial para que los alumnos y los grupos de investigación puedan consultarlos. Las empresas, sobre todo las relacionadas con software, ofrecen temáticas que pueden ser estructuradas como proyectos de investigación.

\section{Portal de conocimiento basado en el mode- lo de gestión}

Con base en el modelo de gestión de conocimiento planteado, se diseñó una página web para la interacción entre la Facultad Tecnológica y las empresas de software6. Mediante el portal (figura 3) es posible que las empresas efectúen búsquedas respecto a proyectos de grado o investigaciones realizadas en la Facultad Tecnológica. Así pues, podría solucionarse uno de los problemas clásicos de las universidades: el desconocimiento de su producción intelectual en el sector productivo. Además, las empresas podrían enterarse de los conocimientos particulares tanto de personas como de colectividades. No obstante, es de notar que al ser un proyecto piloto, la base de datos disponible está restringida -por el momento- y por lo tanto es muy pequeña. Para trabajos futuros será trascendental contar con información más avanzada de toda la universidad y así organizar una base de datos con

6 El modelo fue probado en una primera etapa con varias empresas situadas en Bogotá D.C. Por ejemplo: Disacomputo Ltda., Morris y Aranda Software Corporation. El sitio web del aplicativo es: http://metis.udistrital.edu. co:8095/InteraccionUniversidadEmpresaRM/Home.jsp

REVISTA CIENTÍFICA / ENERO -DICIEMBRE DE 2010 / No. 12 / BOGOTÁ, D.C. 


\section{FIGURA 3. Portal de conocimiento: Facultad Tecnoló-} gica (UD) - empresas de software.

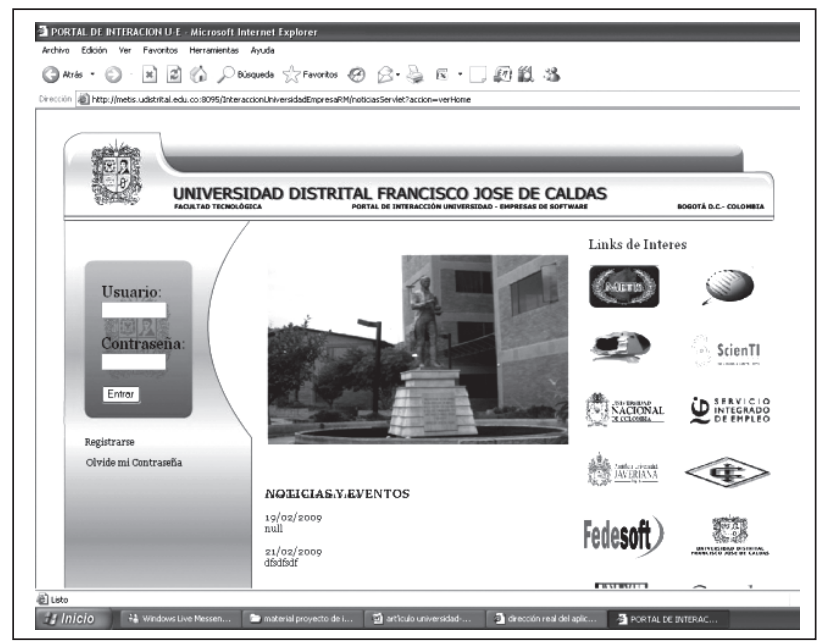

miles de proyectos. Se establece, entonces, que la utilidad práctica del aplicativo está determinada en función del tamaño de la base de datos con el que esté alimentado.

Así mismo, las empresas podrán publicar sus ofertas laborales o investigativas de una manera más concreta y sistemática. Por ejemplo, en la figura 4 aparece una vacante del Gerente de Disacomputo, Carlos Figueroa, en la que se necesitan programadores en J2EE y Java. Cualquier estudiante o investigador interesado que visite el portal podrá enterarse de la oferta publicada y comunicarse personalmente y sin intermediarios (éste tipo de publicación de ofertas puede estar disponible en páginas web institucionales de la universidad). Sin embargo, el valor agregado del portal es su autosuficiencia: funciona desde cualquier lugar del mundo, no toma más de cinco minutos publicar una oferta y puede ser leído por los interesados en tiempo real.

Los comentarios respecto a éste modelo, por parte de los empresarios, se pueden resumir de la siguiente manera:

Como aspectos positivos se resalta la facilidad para conocer digitalmente los trabajos intelectuales desarrollados al interior de la universidad, los cuales,

\section{FIGURA 4. Publicación de una oferta laboral dentro} del portal de conocimiento.

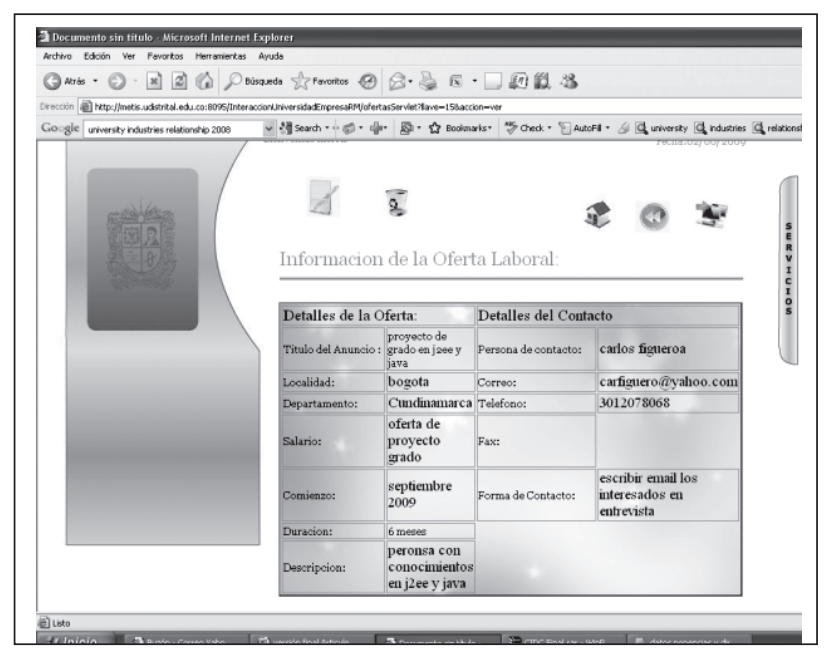

por lo general, son ampliamente desconocidos por el sector productivo. Así mismo, se señala la utilidad de un criterio de búsqueda por tema; al interior de las empresas muchas veces se necesitan personas con conocimientos en temáticas particulares.

Dentro de los aspectos negativos se destacan las restricciones en las bases de datos -el proyecto es piloto. Además, el tamaño de muestra es muy pequeño para una implementación en ambiente real. Se sugiere que en la implementación futura de un sistema más grande se tenga en cuenta la información de toda la universidad discriminada por: facultad, carrera y trayectoria. Por otra parte, se necesitan varios sistemas adicionales como el mantenimiento e ingreso de proyectos nuevos a la base de datos (ésta labor debe ser desarrollada por algún ente institucional al interior de la universidad).

\section{CONCLUSIONES Y RECOMENDACIONES}

En Colombia, la interacción universidad-empresa se encuentra limitada, principalmente, por la diversidad de objetivos de cada parte, lo cual supone un camino sistemático para su unión. El modelo de gestión de conocimiento básico planteado en este documento, por medio de un portal digital, posibilitó la conexión de ofertas y demandas entre 
la Facultad Tecnológica (UD) y algunas empresas de software. De esta manera se logró un acercamiento inicial que le permitió exhibir a la universidad -en este caso al sector del software- sus producciones académicas e investigativas. Por su parte, ésta industria también se benefició con los resultados obtenidos luego de la búsqueda de personal calificado con conocimientos en alguna temática especializada. Sin embargo, dado el carácter del prototipo, es necesario un sistema de base de datos mayor que contenga todos los proyectos desarrollados por la universidad. En un próximo adelanto sobre la versión final del modelo se adicionarán estadísticas de desempeño de proyectos desarrollados en conjunto -mediante la utilización del portal- con el fin de deshabilitar enlaces de menor utilidad e instaurar otros que incrementen la interacción con las empresas.

\section{BIBLIOGRAFÍA}

- Vargas, M.; Malaver, F. y Zerda, A. (2003), La innovación tecnológica en la industria colombiana, Bogotá D.C., Colciencias.

- Porter, M. (1987), Ventaja competitiva. Creación $y$ sostenimiento de un desempeño superior, México D.F., Cecsa.

- Corona, L. y Jasso, J. (2008, 22 de septiembre), "Technology transfer from UNAM 'S Research Centers to Industry: impacts of its organization and knowledge profile", VI Globelics Conference, México D.F.

- Karthikeyan, S. (2008), "Effective industry Institute Interaction Through SIG for the improvement of placement activities in Engineering Education: A case study of Thiagarajar College of Engineering", en Asian Journal of Information Technology, pp. 272-276.- Geuna, A. y Martin, B. (2003), University Research Evaluation and Funding: An International Comparison, Minerva 41, pp. 277-304.
- Manjarrés, L. (2008), "Coexistence of University-Industry relations and academic research: Barrier to or incentive for scientific productivity", en Scientometrics, vol. 76, núm.3, pp. 561-576.

- Warner, A. (2000), "Economic Creativity." en The Global Competitiveness Report, World Economic Forum, New York, Oxford University Press.

- Porter, M. (1998, noviembre-diciembre), "Clusters and the new economics of competition", en Harvard Business Review, pp. 77-90.

- Cepero, M. (2007), Strategies for the development of the software industry en Colombia [tesis de maestría], Maestría en Gestión de Tecnología. Massachusetts Institute of Technology (MIT), Cambridge, Massachusetts.

- Nezu, R. (2005), Technology transfer, intellectual property and effective university-industry partnerships. the experience of China, India, Japan, Phillipines, the Republic of Korea, Singapore and Thailan, Génova, World Intellectual Property Organization.

- Colombia, Cámara de Comercio de Bogotá (2005), Balance Tecnológico cadena productiva desarrollo de software en Bogotá y Cundinamarca, Departamento de publicaciones Cámara de Comercio de Bogotá.

- Nonaka, I. y Takeuchi, H. (1995), The knowledge creating company, New York, Oxford University Press.

- Krogh, G.; Ichijo, K. y Nonaka, I., (2000), How to unlock the mistery of tacit knowledge and release the power of innovation, New York, Oxford University Press. 